\title{
Charge Transport in Non-Irradiated and Irradiated Silicon Diodes
}

\author{
C. Leroy $^{1}$, P. Roy ${ }^{1}$, G. Casse ${ }^{2}$, M. Glaser ${ }^{2}$, E. Grigoriev ${ }^{2}$, F. Lemeilleur ${ }^{2}$ \\ ${ }^{1}$ Université de Montréal, Montréal (Québec) H3C 3J7, Canada \\ ${ }^{2}$ CERN, Geneva, Switzerland
}

\begin{abstract}
A model describing the transport of charge carriers generated in silicon detectors (standard planar float zone and MESA diodes) by ionizing particles is presented. The current pulse response induced by $\alpha$ and $\beta$ particles in non-irradiated detectors and detectors irradiated up to fluences $\Phi \approx 3 \cdot 10^{14}$ particles $/ \mathrm{cm}^{2}$ is reproduced through this model: i) by adding a small n-type region $15 \mu \mathrm{m}$ deep on the $p^{+}$side for the standard planar float zone detectors at fluences beyond the $\mathrm{n}$ to $\mathrm{p}$-type inversion and ii) for the MESA detectors, by considering one dead layer $14 \mu \mathrm{m}$ deep (observed experimentally) on each side, and introducing a second (delayed) component. For both types of detectors, the model gives mobilities decreasing linearily up to fluences of about $5 \cdot 10^{13}$ particles $/ \mathrm{cm}^{2}$ and converging, beyond, to saturation values of about $1000 \mathrm{~cm}^{2} / \mathrm{Vs}$ and 455 $\mathrm{cm}^{2} / \mathrm{Vs}$ for electrons and holes, respectively. At a fluence $\Phi \approx 10^{14}$ particles $/ \mathrm{cm}^{2}$, charge collection deficits of about $13 \%$ for $\beta$ particles, $25 \%$ for $\alpha$ particles incident on the front and $35 \%$ for $\alpha$ particles incident on the back of the detector are found for both type of diodes.
\end{abstract}

\section{INTRODUCTION}

The electrical characteristics of n-type silicon detectors (standard planar float zone (SPFZ) and MESA) as a function of the particle fluence $(\Phi)$ can be extracted by modelling the transport of the carriers of the charge generated by $\alpha$ or $\beta$ particles in non-irradiated and irradiated silicon detectors. The model is used to fit the experimental signal-current pulse responses (measured as a function of the collection time) induced by $\alpha$ and $\beta$ particles in $p^{+}-n-n^{+}$diodes. The extracted electrical characteristics of a $p^{+}-n-n^{+}$diode are the effective impurity or dopant concentration $\left(N_{e f f}\right)$, the electron $\left(\mu_{e}\right)$ and hole $\left(\mu_{h}\right)$ mobilities, and the charge carrier lifetimes $\left(\tau_{t e}, \tau_{t h}\right)$.

\section{THE CHARGE TRANSPORT MODEL}

The electrical characteristics are extracted from a system of five partial differential equations: the current continuity equations for electrons and holes, the Poisson equation (which determines the electric field and considers the plasma effect) and two equations relating the concentration of trapped to the untrapped charges. No analytical solution can be obtained, so the equations are discretized using Gummel's decoupling scheme [1] to obtain a numerical solution. The observed signal $(V(t))$ is a convolution of the current $(I(t)$ obtained from Ramo's theorem [2]) produced by all the individual charge carriers and the response from the system, which is simply an RC circuit. The response of the system is Gaussian with a characteristic time constant $\sigma=R_{a} C$, where $C$ is the capacitance of the detector and $R_{a}=50 \Omega$ the input impedance of the amplifier:

$$
\begin{aligned}
I(t) & =\frac{18 D_{a} t+r_{0}^{2}}{w r_{0}^{2}} \int_{0}^{w}\left(\mu_{e} n+\mu_{h} p\right) E d x \\
V(t) & =\frac{G R_{a}}{\sigma \sqrt{2 \pi}} \sum_{e, h} \int_{-\infty}^{\infty} I\left(t^{\prime}\right) \exp \left(-\frac{\left(t-t^{\prime}\right)^{2}}{2 \sigma^{2}}\right) d t^{\prime}
\end{aligned}
$$

where $n$ and $p$ are the concentration of electrons and holes, $E$ the electric field, $D_{a}$ the ambipolar diffusion constant, $G=1000$ the gain of the amplifier, $r_{0}$ the initial radius of the column of deposited charge and $w$ the thickness of the diodes. The drift velocity of the charge carrier reaches a saturation value $v_{s}$ for electric field values around $10^{4} \mathrm{~V} / \mathrm{cm}$. The empirical equation describing the mobility as a function of the electric field is [3]:

$$
\mu(x)=\frac{\mu_{0}}{\left[1+\left(\mu_{0} E(x) / v_{s}\right)^{m}\right]^{1 / m}}
$$

where $\mu_{0}$ is the zero field mobility, $m=1$ for holes, $m=2$ for electrons; $v_{s}=1.05 \cdot 10^{7}$ and $10^{7} \mathrm{~cm} / \mathrm{s}$ for electrons and holes, respectively.

The mobilities are also dependent on the temperature and dopant concentrations. Those dependences are taken into account via the empirical equation [4]:

$$
\mu\left(T, N_{e f f}\right)=\mu_{\min }+\frac{\mu_{0}\left(\frac{T}{300}\right)^{\nu}-\mu_{\min }}{1+\left(\frac{T}{300}\right)^{\xi}\left(\frac{N_{e f f}}{N_{\text {ref }}}\right)^{\alpha}}
$$

where the values used for the electrons (holes) are: $\mu_{\min }=55.24$ (49.7) $\mathrm{cm}^{2} / \mathrm{Vs}, N_{\text {ref }}=1.072 \cdot 10^{17}\left(1.606 \cdot 10^{17}\right)$ dopants $/ \mathrm{cm}^{3}, \nu$ $=-2.3(-2.2), \xi=-3.8(-3.7), \alpha=0.73(0.70), T$ is the temperature in Kelvin and $\mu_{0}$ is the mobility at $T=300 \mathrm{~K}$.

The quantities of interest are extracted by using the code MINUIT [5] to minimize the $\chi^{2}$ obtained from fitting the numerical solution found for $\mathrm{V}(\mathrm{t})$ to the measured current pulse response induced by $\alpha$ and $\beta$ particles.

Electrons from a ${ }^{106} \mathrm{Ru}$ source with an energy $>2 \mathrm{MeV}$, selected by an external trigger, and $\alpha$ particles from a ${ }^{241} \mathrm{Am}$ source with an energy of $5.49 \mathrm{MeV}$ were used. The current pulses induced by particles penetrating the silicon diode are detected by a fast current amplifier. The pulses are recorded by a digital oscilloscope used in averaging mode, to improve the signal-to-noise ratio. The diodes studied in the present work are listed in Table 1 with their thickness and resistivity. 
Table 1

Characteristics of the studied diodes. The detectors were irradiated (column 7) by step of fluence up to $9.92 \cdot 10^{13} \mathrm{n} / \mathrm{cm}^{2}$ for $\mathrm{M}_{4}, \mathbf{7 . 4 8} \cdot 10^{13}$ $\mathrm{p} / \mathrm{cm}^{2}$ for M18, M25, M35, and up to $2.87 \cdot \mathbf{1 0}^{\mathbf{1 4}} \mathrm{p} / \mathrm{cm}^{2}$ for P44, P86, P88, P135, P189, P300 and P304. M49, M50 and M53 were not irradiated

\begin{tabular}{|l|l|l|l|l|l|l|}
\hline Detector & Process & $\begin{array}{l}\text { Current } \\
\text { pulse source }\end{array}$ & $\begin{array}{l}\text { Thickness } \\
(\mu \mathrm{m})\end{array}$ & $\begin{array}{l}\mathrm{N}_{e f f}(\Phi=\mathbf{0}) \\
\left(10^{11} \mathrm{~cm}^{-3}\right)\end{array}$ & $\begin{array}{l}\text { Resistivity } \\
\mathrm{k} \Omega \cdot \mathrm{cm}\end{array}$ & $\begin{array}{l}\text { Maximum fluence } \\
\left(10^{13} \mathrm{~cm}^{-2}\right)\end{array}$ \\
\hline M4 & SPFZ & $\alpha$ & 317 & -3.4 & 12.2 & $9.92 \mathrm{n}$ \\
\hline M18 & SPFZ & $\alpha, \beta$ & 309 & -4.1 & 11. & $7.48 \mathrm{p}$ \\
\hline M25 & SPFZ & $\alpha, \beta$ & 308 & -2.1 & 23. & $7.48 \mathrm{p}$ \\
\hline M35 & SPFZ & $\alpha$ & 508 & -1.7 & 24. & $7.48 \mathrm{p}$ \\
\hline M49 & SPFZ & $\beta$ & 301 & -4.7 & 8.9 & - \\
\hline M50 & SPFZ & $\beta$ & 471 & -1.8 & 22.8 & - \\
\hline M53 & SPFZ & $\beta$ & 223 & -5.4 & 7.7 & - \\
\hline P44 & MESA & $\alpha, \beta$ & 306 & -18 & 2.0 & $28.7 \mathrm{p}$ \\
\hline P86 & SPFZ & $\alpha, \beta$ & 290 & -21 & 2.5 & $28.7 \mathrm{p}$ \\
\hline P88 & SPFZ & $\alpha, \beta$ & 290 & -19 & 2.5 & $28.7 \mathrm{p}$ \\
\hline P135 & MESA & $\alpha, \beta$ & 308 & -17 & 2.0 & $28.7 \mathrm{p}$ \\
\hline P189 & SPFZ & $\alpha, \beta$ & 294 & -21 & 2.5 & $28.7 \mathrm{p}$ \\
\hline P300 & MESA & $\alpha, \beta$ & 303 & -6 & 6.0 & $28.7 \mathrm{p}$ \\
\hline P304 & SPFZ & $\alpha, \beta$ & 320 & -6 & 6.0 & $28.7 \mathrm{p}$ \\
\hline
\end{tabular}

\section{STANDARD PLANAR FLOAT ZONE DIODES}

Fits of the charge carriers transport model to the current pulses induced by relativistic electrons and by $\alpha$-particle either on the front-side or the back-side of non-irradiated SPFZ detectors (represented in Fig. 1) reproduces well the shape of the measured current pulses $(\alpha$ and $\beta$ ). Figure 2 shows the contribution of the electrons and holes to the signal.

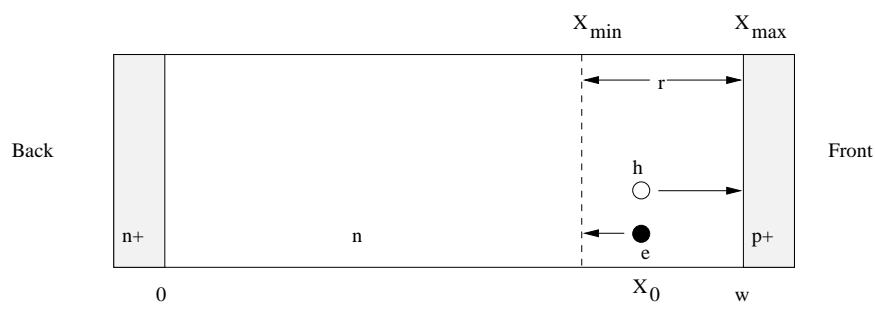

Figure 1: Schematic representation of a SPFZ diode.

Table 2 shows the initial mobilities at $\Phi=0$ of the diodes obtained from the model. The average mobilities achieved for electrons and holes are: $\mu_{e}=1284 \pm 21 \mathrm{~cm}^{2} / \mathrm{Vs}$ and $\mu_{h}=$ $482 \pm 10 \mathrm{~cm}^{2} / \mathrm{Vs}$, respectively.

The detectors have been irradiated either with $\approx 1 \mathrm{MeV}$ neutrons at CERN-PSAIF, up to a fluence of $9.92 \cdot 10^{13} \mathrm{n} / \mathrm{cm}^{2}$ or with $24 \mathrm{GeV} / \mathrm{c}$ protons at CERN-PS, up to a fluence of $2.87 \cdot 10^{14} \mathrm{p} / \mathrm{cm}^{2}$ (column 7 in Table 1 ).

The results of the charge transport model fits to the experimental data permit the extraction of the value of $N_{e f f}$ as a function of the fluence:

$$
N_{e f f}=-N_{d} \exp (-c \Phi)+N_{a}+b \Phi
$$

where $N_{d}$ and $N_{a}$ are the concentration of donnors and acceptors at $\Phi=0$, respectively; $b$ and $c$ are the acceptor creation and donnor removal parameters, respectively. By
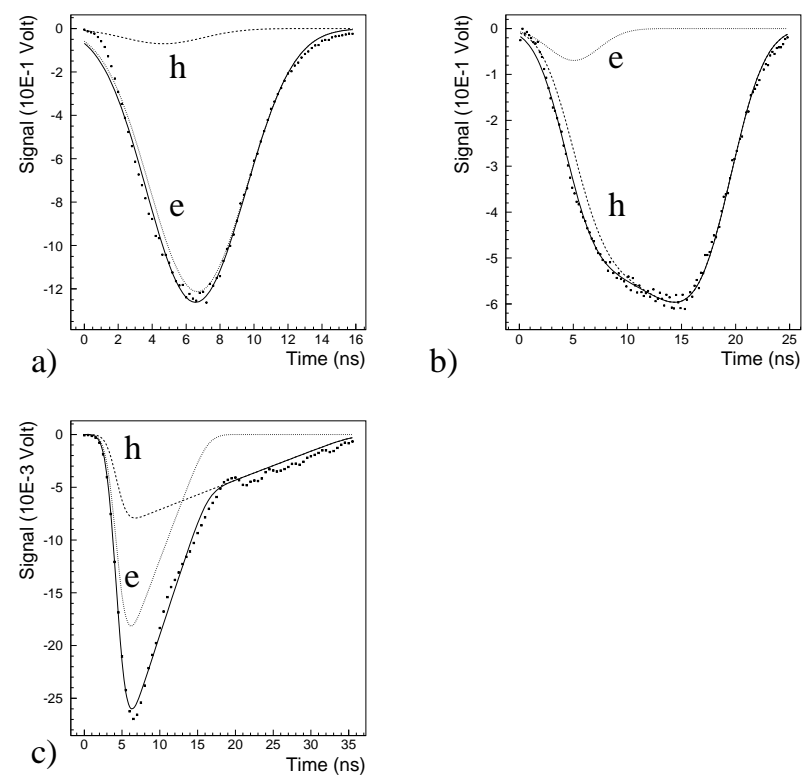

Figure 2: Fits (full line) of the charge transport model to the current pulse response induced at $\Phi=0$ by $\alpha$ particles incident on the front side (a), on the back side (b) of detector M25 and by relativistic electrons on detector M50 (c); A bias voltage $V_{b}=160 \mathrm{~V}$ is applied in all cases. The individual electrons (e) and holes (h) contributions are shown.

using Eq. (5) to describe the evolution of $N_{\text {eff }}$ with fluence, one obtains the results shown in Fig. 3 for two detectors (P86 and P88). The values of the parameters obtained using Eq. (5) for all the detectors are reported in Table 3.

Using a simple $\mathrm{pn}^{+}$junction after the $\mathrm{n}$ to $\mathrm{p}$-type inversion (which takes place at a fluence $\Phi \equiv \Phi_{\text {inv }} \approx 5 \cdot 10^{12}$ particles $/ \mathrm{cm}^{2}$, a detector with an higher initial resistivity inverts from $\mathrm{n}$ to $\mathrm{p}$-type at lower fluence value), the charge 
Table 2

Mobilities at $\Phi=0$ of the SPFZ diodes as obtained from the model

\begin{tabular}{|l|l|l|}
\hline Detector & $\begin{array}{l}\mu_{h} \\
\left(\mathrm{~cm}^{2} / \mathrm{Vs}\right)\end{array}$ & $\begin{array}{l}\mu_{e} \\
\left(\mathrm{~cm}^{2} / \mathrm{Vs}\right)\end{array}$ \\
\hline M4 & $504 \pm 2$ & $1278 \pm 15$ \\
\hline M18 & $474 \pm 2$ & $1236 \pm 15$ \\
\hline M25 & $476 \pm 2$ & $1308 \pm 28$ \\
\hline M35 & $472 \pm 3$ & $1272 \pm 5$ \\
\hline M49 & $546 \pm 11$ & $1266 \pm 24$ \\
\hline M50 & $529 \pm 13$ & $1272 \pm 20$ \\
\hline M53 & $478 \pm 12$ & $1350 \pm 20$ \\
\hline P88 & $459 \pm 4$ & $1222 \pm 20$ \\
\hline P189 & $480 \pm 20$ & $1340 \pm 27$ \\
\hline P304 & $495 \pm 3$ & $1124 \pm 22$ \\
\hline
\end{tabular}
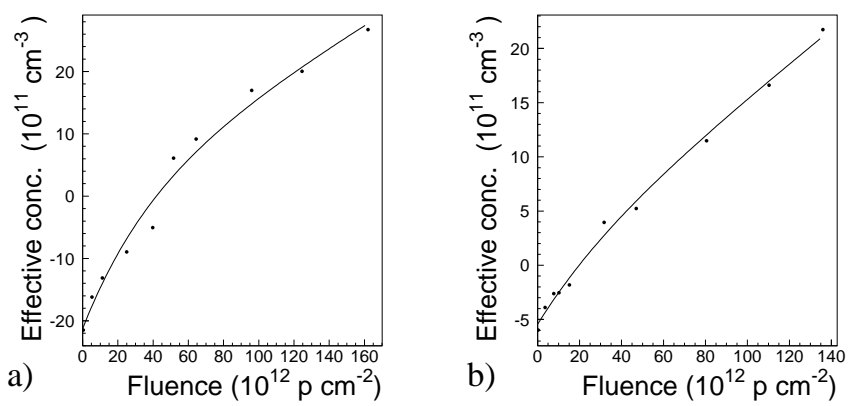

Figure 3: Evolution of the effective concentration of dopants as a function of fluence for the detectors a) P86 and b) P304.

Table 3

Values of the parameters obtained to describe the evolution of the effective concentration with fluence

\begin{tabular}{|l|c|c|c|c|}
\hline Det. & $\begin{array}{c}N_{d} \\
\left(10^{11} / \mathrm{cm}^{3}\right)\end{array}$ & $\begin{array}{c}N_{a} \\
\left(10^{11} / \mathrm{cm}^{3}\right)\end{array}$ & $\begin{array}{c}b \\
\left(10^{-2} / \mathrm{cm}\right)\end{array}$ & $\begin{array}{c}c \\
\left(10^{-13} \mathrm{~cm}^{2}\right)\end{array}$ \\
\hline M4 & 3.1 & 0 & 1.5 & 8.1 \\
\hline M18 & 3.7 & 0 & 2.8 & 12. \\
\hline M25 & 2.0 & 0 & 2.5 & 21. \\
\hline M35 & 36. & 35. & 0.2 & 1.0 \\
\hline P44 & 17. & 0 & 0.9 & 2.4 \\
\hline P86 & 33. & 11. & 1.0 & 2.2 \\
\hline P88 & 30. & 11. & 1.5 & 1.5 \\
\hline P135 & 23. & 7.0 & 0.7 & 1.3 \\
\hline P189 & 19. & 0 & 1.4 & 3.2 \\
\hline P304 & 5.4 & 0 & 1.6 & 2.9 \\
\hline
\end{tabular}

carriers transport model poorly reproduces the measured $\mathrm{V}(\mathrm{t})$ as a function of the collection time for $\Phi>\Phi_{i n v}$. Also, the mobilities keep on decreasing continuously with increasing fluence. For instance, while $\mu_{h}=474.4 \mathrm{~cm}^{2} / \mathrm{Vs}$ and $\mu_{e}=1236 \mathrm{~cm}^{2} / \mathrm{Vs}$ for the detector M18 at $\Phi=0$, the values of the mobilities for this detector drop to $\mu_{h}=338 \mathrm{~cm}^{2} / \mathrm{Vs}$ and $\mu_{e}=700 \mathrm{~cm}^{2} / \mathrm{Vs}$ at a fluence of $\Phi=7.48 \cdot 10^{13} \mathrm{p} / \mathrm{cm}^{2}$ without showing any sign of saturation.

In order to fit the data and to account for the evolution of the electrical characteristic of the detectors with fluence beyond $\Phi_{i n v}$, the electric field is modified after inversion by introducing a n-type region $15 \mu \mathrm{m}$ deep near the $p^{+}$contact. This concept of double junction can be also found in [6] and in other references contemporary with the present work $[7,8]$. This modification of the electric field permits to reproduce the measured $\mathrm{V}(\mathrm{t})$ as a function of the collection time for $\Phi>\Phi_{i n v}$, as shown in Fig. 4. The evolution of the pulse shape of one detector (M25) as a function of fluence, as described by applying the model, is shown in Fig. 5 through Fig. 7.
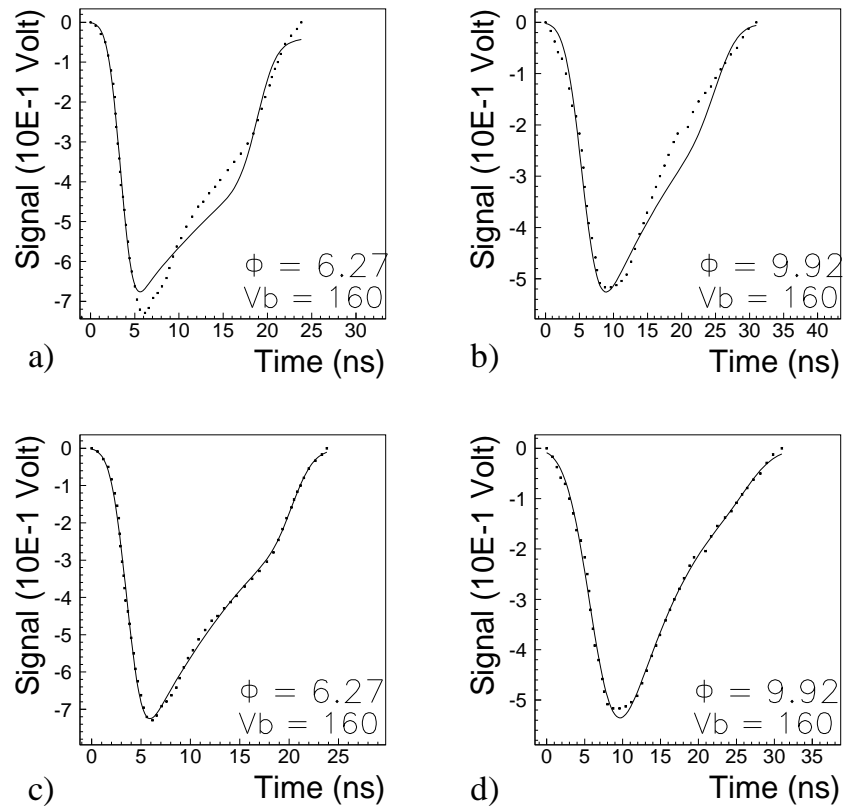

Figure 4: Fits (full line) for detector M4 before ( $a$ and b) and after (c and d) the introduction of the $15 \mu \mathrm{m}$ n-type junction on the $p^{+}$side after inversion at fluences of $\Phi=6.27(\mathrm{a}, \mathrm{c})$ and $9.92 \cdot 10^{13} \mathrm{n} / \mathrm{cm}^{2}$ $(\mathrm{b}, \mathrm{d})$ for an $\alpha$ particle on the back side with an applied voltage of $V_{b}=160$ Volts.
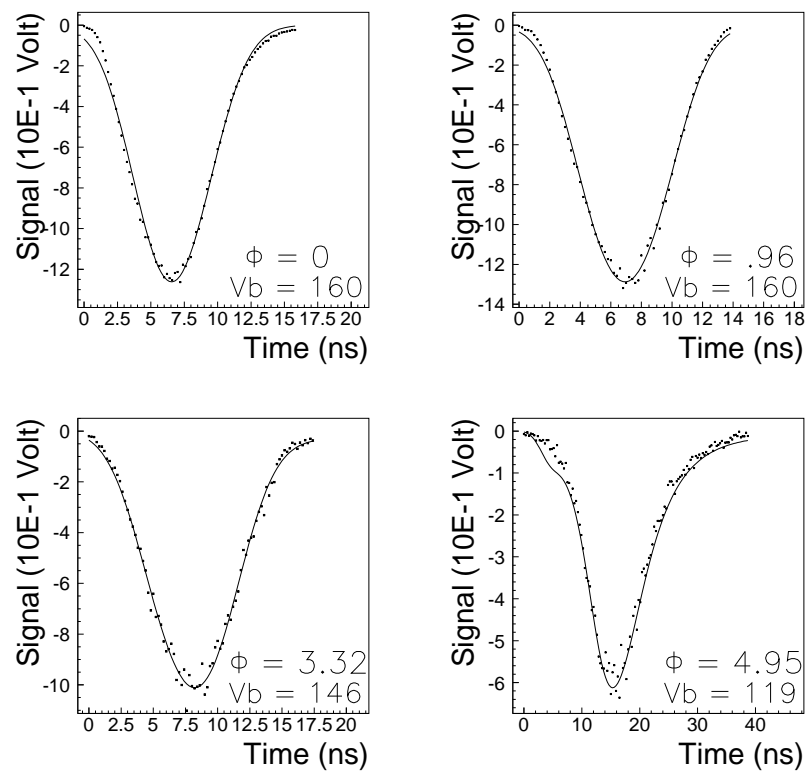

Figure 5: Fits (full line) of the current pulse response induced by $\alpha$ particles incident on the front side of detector M25 for successive levels of fluence $\Phi$ from 0 up to $4.95 \cdot 10^{13} \mathrm{p} / \mathrm{cm}^{2}$ ( $\Phi$ is in units of $10^{13} \mathrm{p} / \mathrm{cm}^{2}, V_{b}$ is the applied voltage in volts). 

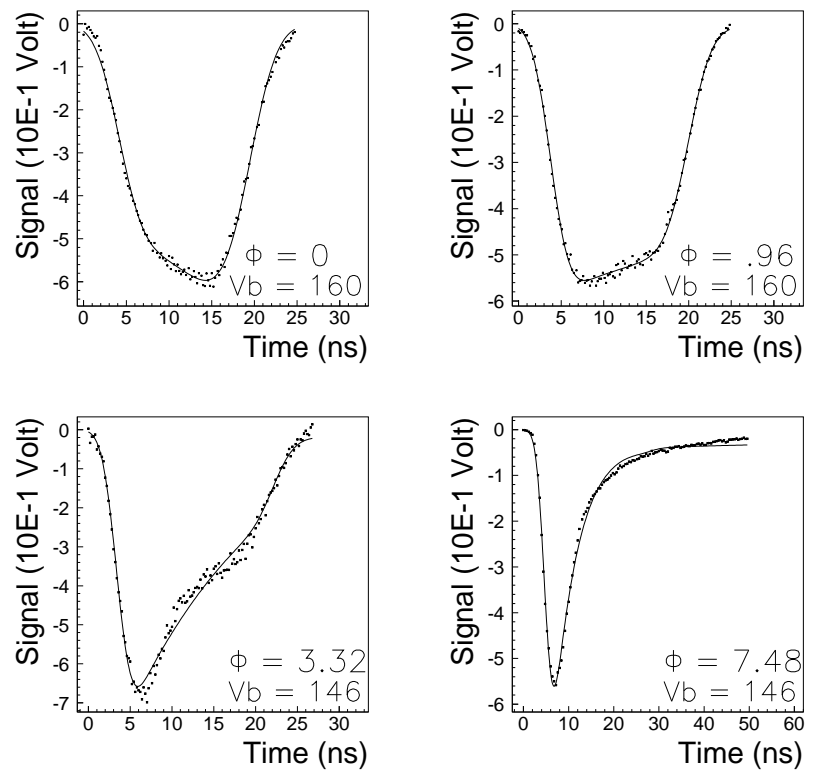

Figure 6: Fits (full line) of the current pulse response induced by $\alpha$ particles incident on the back side of detector M25 for successive levels of fluence $\Phi$ from 0 up to $7.48 \cdot 10^{13} \mathrm{p} / \mathrm{cm}^{2}$ ( $\Phi$ is in units of $10^{13} \mathrm{p} / \mathrm{cm}^{2}, V_{b}$ is the applied voltage in volts).
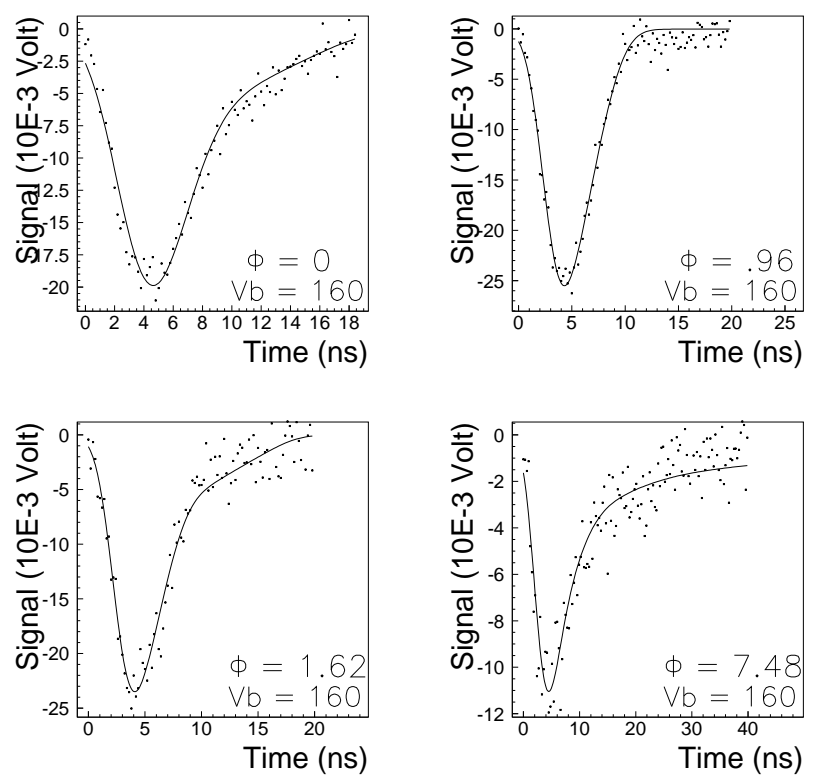

Figure 7: Fits (full line) of the current pulse response induced by $\beta$ particles incident on detector M25 for successive levels of fluence $\Phi$ from 0 up to $7.48 \cdot 10^{13} \mathrm{p} / \mathrm{cm}^{2}$ ( $\Phi$ is in units of $10^{13} \mathrm{p} / \mathrm{cm}^{2}, V_{b}$ is the applied voltage in Volts).

The behaviour of the mobility with fluence is also modified by the introduction of the $15 \mu \mathrm{m}$ n-type region. As it can be seen from Fig.8, the mobility now tends, after an initial decrease, towards the saturation values $\mu_{\text {sat }, e} \approx 1000 \mathrm{~cm}^{2} / \mathrm{Vs}$ and $\mu_{s a t, h} \approx 455 \mathrm{~cm}^{2} / \mathrm{Vs}$ for the electrons and holes for $\Phi>5 \cdot 10^{13}$ particles $/ \mathrm{cm}^{2}$, respectively. This figure also shows that the mobility values obtained using either $\alpha$ or $\beta$ particles data are in agreement, which provides a consistency check of the model.
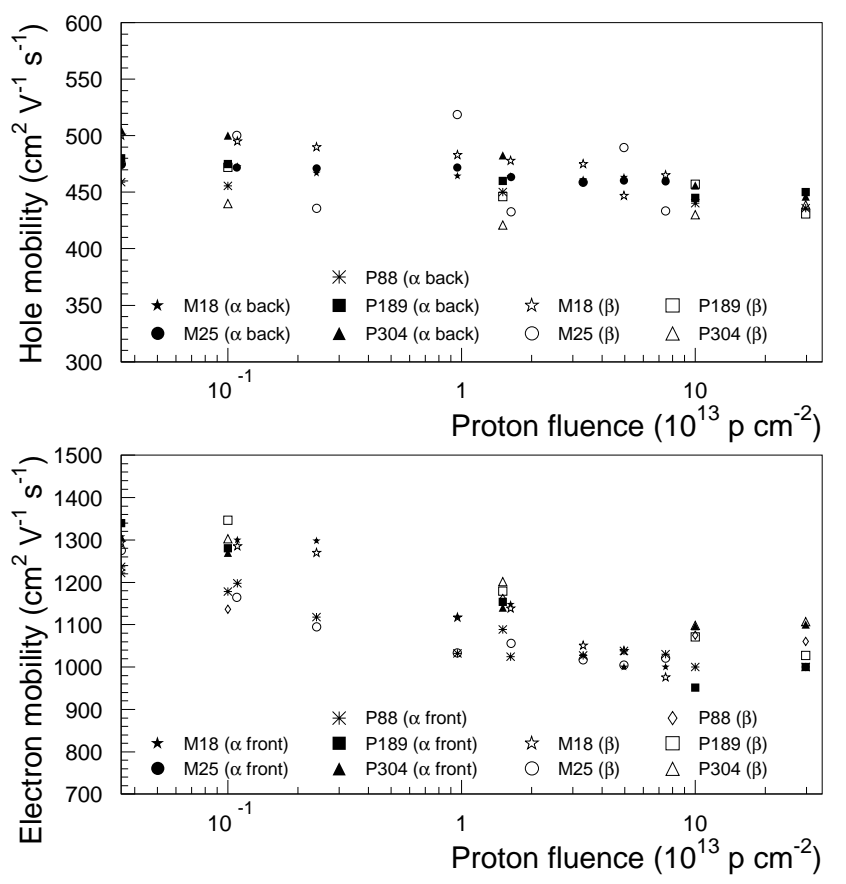

Figure 8: Evolution of the mobilities as a function of fluence for SPFZ diodes, as extracted from the model using $\alpha$ front, $\alpha$ back and $\beta$ particles data.

\section{MESA DIODES}

The MESA process for building diodes is an alternative to the planar process that is simpler and cheaper, as it involves no oxidation or masking. Studies [9] have shown that a layer of about 14 microns on each side of the diode acts as a dead layer. So by taking this feature into account, it is possible to adapt the model, successfully applied to SPFZ detectors, to the description of the MESA detectors.

We start with a MESA detector of thickness $w$, of which a thickness $X_{\text {dead }}$ is considered dead on each side as shown in Fig. 9. We can define the active thickness of the detector as $w^{\prime}=w-2 \cdot X_{\text {dead }}$.

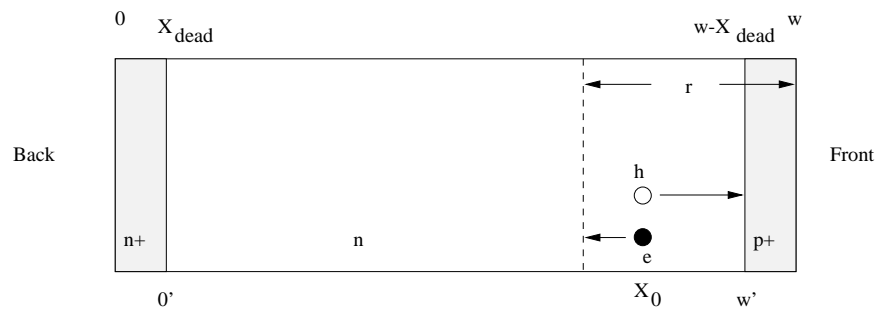

Figure 9: Schematic representation of a MESA diode with the dead layers on each side.

The initial distribution of charge carriers at time $t=0$ over the total thickness $w$ is the same as for a SPFZ detector, even though only the active region $X_{\text {dead }}<x<w-X_{\text {dead }}$ (i.e. $0^{\prime}<x<w^{\prime}$ ) will generate the signal. This modification alone to the model (line 1 in Fig. 10a) is not enough as the measured current-pulses are wider. We consider that a fraction $(f)$ of the electron-hole pairs created in the dead zones becomes active only 
at a later time $T_{\text {late }}$ which gives a second component (line 2 in Fig. 10b). A very good description of the measured current-pulse of a MESA diode is obtained by adding the two components (line 3 in Fig. 10b).
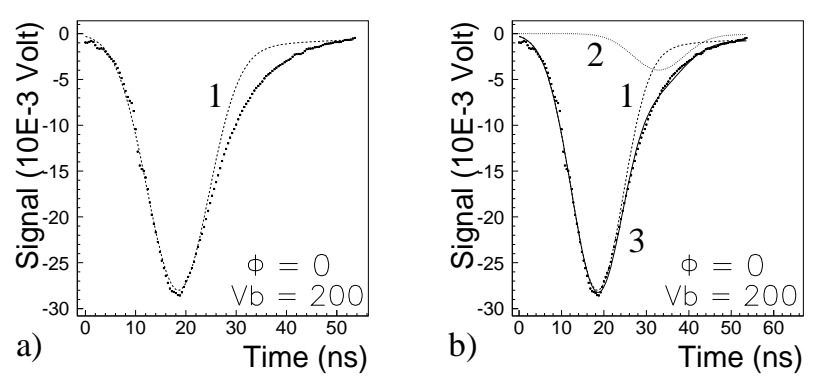

Figure 10: Current pulse response induced by $\alpha$ particles incident on the back of a MESA diode before (line 1) and after (line 3 ) the addition of a second (delayed) component (line 2) at $\Phi=0$ and applied voltage $V_{b}=200$ Volts.

For $\alpha$ particles incident on the front side of the detector, some electrons will be released near the front (see Fig. 11a):

$$
n\left(x, T_{\text {late }}\right)=n\left(x, T_{\text {late }}\right)+f
$$

for $\alpha$ particles incident on the back side of the detector, some holes will be released near the back (see Fig. 11b):

$$
p\left(x, T_{\text {late }}\right)=p\left(x, T_{\text {late }}\right)+f
$$

or a combination of both cases for $\beta$ particles (see Fig. 11c).

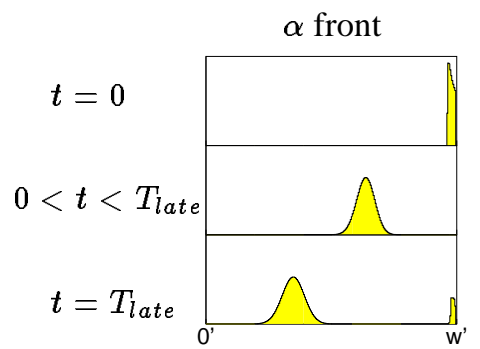

$\alpha$ back
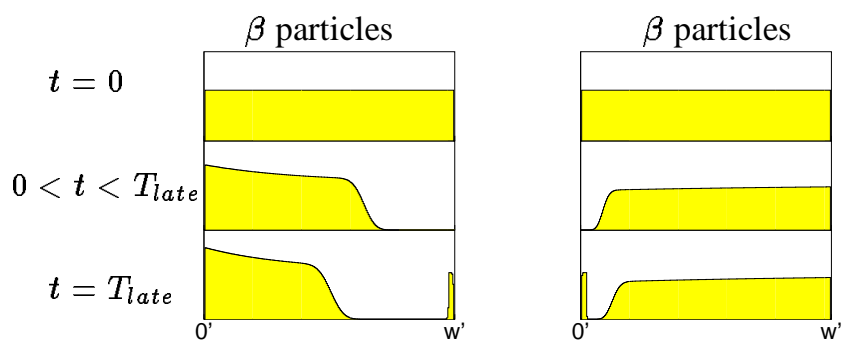

Figure 11: Representation of the distribution of charge carriers in a MESA diode before and after the inclusion of the second component.

Fits of the model to the current-pulse response of MESA detector P135 induced by $\alpha$ and $\beta$ particles, taking into account the second (delayed) component, are shown in Fig. 12 as a function of fluence. The mobilities as a function of fluence for MESA diodes are shown in Fig. 13 and are in agreement with the values obtained for the SPFZ detectors.
Table 4

Mobilities at $\Phi=0$ of the MESA diodes as obtained from the model

\begin{tabular}{|l|l|l|}
\hline Detector & $\begin{array}{l}\mu_{h} \\
\left(\mathrm{~cm}^{2} / \mathrm{Vs}\right)\end{array}$ & $\begin{array}{l}\mu_{e} \\
\left(\mathrm{~cm}^{2} / \mathrm{Vs}\right)\end{array}$ \\
\hline P44 & $455 \pm 15$ & $1422 \pm 24$ \\
\hline P135 & $472 \pm 9$ & $1310 \pm 23$ \\
\hline P300 & $469 \pm 12$ & $1298 \pm 18$ \\
\hline
\end{tabular}
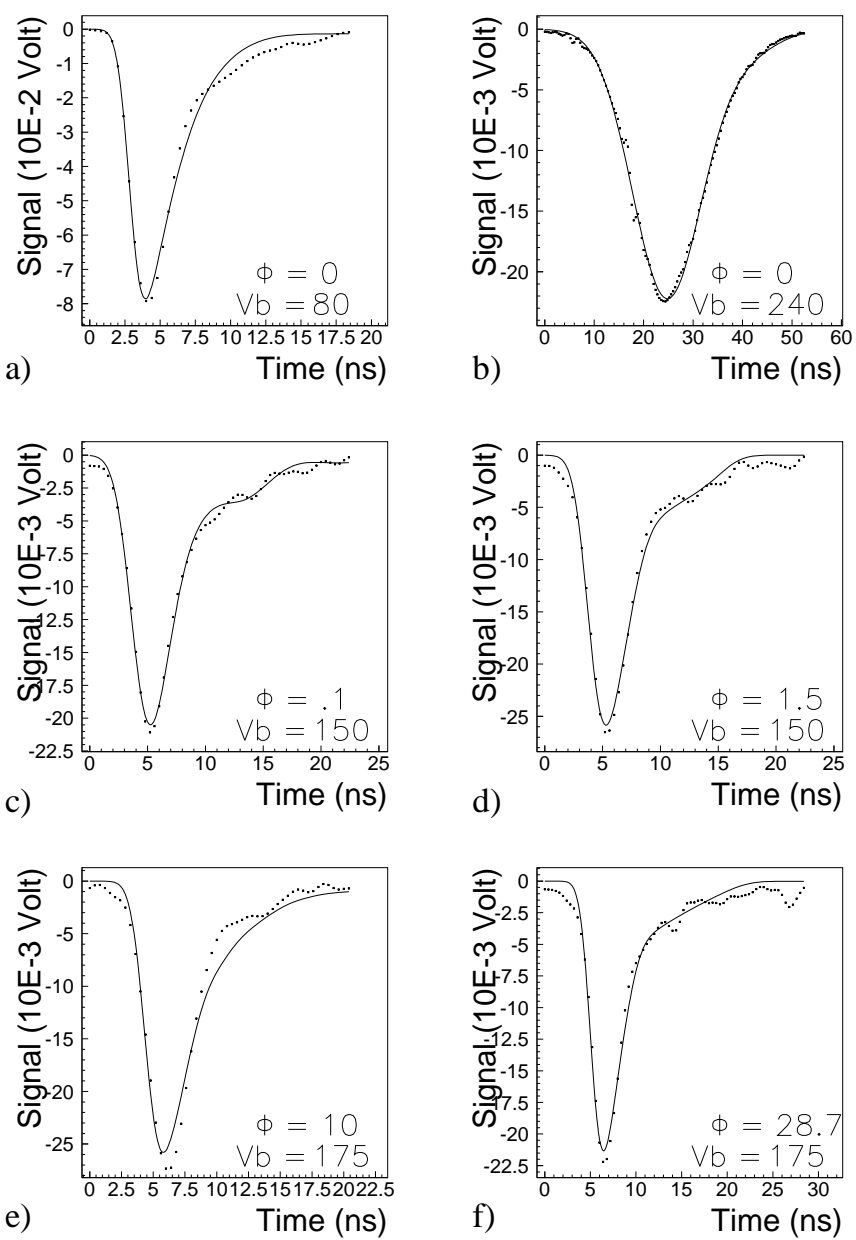

Figure 12: Fits (full line) of the current pulse response induced by $\alpha$ particles incident on the front side (a), on the back side (b) and for $\beta$ particles (c-f) of MESA detector P135 for successive levels of fluence $\Phi$ from 0 up to $2.87 \cdot 10^{14} \mathrm{p} / \mathrm{cm}^{2}$ ( $\Phi$ is in units of $10^{13} \mathrm{p} / \mathrm{cm}^{2}, V_{b}$ is the applied voltage in volts).

\section{Charge COLlection EFFICIENCY}

Since the number of defects increases as the fluence gets higher, the charge carrier lifetimes due to trapping decreases. The integration of the signal over the collection time allows the determination of the collected charge. Thus, a comparison between the results obtained using the trapping lifetime extracted at a certain fluence, with those obtained if no trapping had occurred, allows the calculation of the charge collection efficiency (CCE). As it can be seen from Fig 14a and 14b, for neutron (proton) irradiated SPFZ detectors, a charge collection deficit around $12 \%(25 \%)$ is calculated for $\alpha$ 

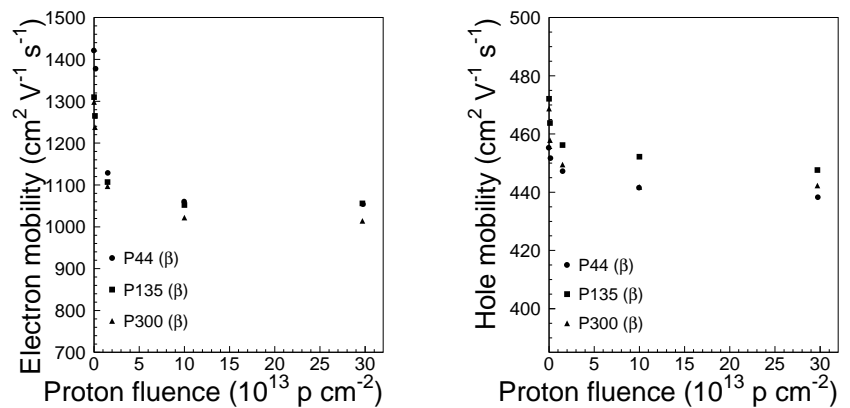

Figure 13: Evolution of the mobilities as a function of fluence for MESA diodes.

particles incident on the front side and about $18 \%(35 \%)$ for $\alpha$ particles incident on the back side of the detector, for a fluence of $\approx 10^{14}$ particles $/ \mathrm{cm}^{2}$. Direct measurements [10] of CCE using $\alpha$ particles from a Th $C$ ' source with an energy of $8.78 \mathrm{MeV}$ on detectors irradiated up to a fluence of $\approx 10^{14}$ protons $/ \mathrm{cm}^{2}$ show a smaller deficit $(\approx 5 \%$ on the front side and $\approx 10 \%$ on the back side). Those discrepancies can be explained. First, an $\alpha$ particle of 5 (8.78) $\mathrm{MeV}$ has a range of $\approx 25$ (57) microns in silicon, most of the energy being deposited toward the end of the path. We can assume that most electron-hole pairs are created around 20 (50) microns from the surface. Thus, for a typical detector of 300 microns, the charge carriers generated by $\alpha$ particles from an ${ }^{241} \mathrm{Am}$ source will experience more trapping as they spend $\approx 15 \%$ more time in the detector than those generated by $\alpha$ particles from a Th C' source. Secondly, the setup used with the Th C' source in ref. [10] had a shaping time $(1 \mu \mathrm{s})$ larger than the shaping time (100 ns) used with the present ${ }^{241}$ Am source setup. A large shaping time means that the trapped charges are more likely to untrap and thus reduce the observed charge collection deficit. For $\beta$ particles, a collection deficit of about $13 \%$ is calculated (Fig. 14c) for a fluence of $\approx 10^{14}$ particles $/ \mathrm{cm}^{2}$. This latter result is in agreement with the $12 \%$ deficit obtained from direct charge collection efficiency measurements made with $\beta$ particles (shaping time of $100 \mathrm{~ns}$ ) [11] for detectors irradiated at fluences $\approx 10^{14}$ particles $/ \mathrm{cm}^{2}$. For MESA detectors, using a signal induced by $\beta$ particles, charge collection deficits around $12 \%$ and $18 \%$ are calculated (Fig. $14 \mathrm{~d}$ ) for fluences of $\approx 1$ and $3 \cdot 10^{14}$ protons $/ \mathrm{cm}^{2}$, respectively. This result is similar to the one obtained for SPFZ detectors at a fluence of $\approx 10^{14}$ protons $/ \mathrm{cm}^{2}$.

\section{CONCLUSIONS}

The present study brings the following conclusions: The model describing the transport of the carriers of charge generated in silicon detectors by ionizing particles allows one to reproduce the current pulse response of non-irradiated and irradiated SPFZ detectors induced by $\alpha$ and $\beta$ particles up to fluences around $\mathrm{n}$ to p-type inversion $\left(\Phi \approx \Phi_{\text {inv }}\right)$ using a simple $p^{+}-n-n^{+}$diode. Beyond inversion $\left(\Phi>\Phi_{\text {inv }}\right)$ a small n-type region $15 \mu \mathrm{m}$ deep is introduced on the $p^{+}$side of the detector. The introduction of this region modifies the electric field after inversion and permits the charge carriers transport model to reproduce the experimental data up to
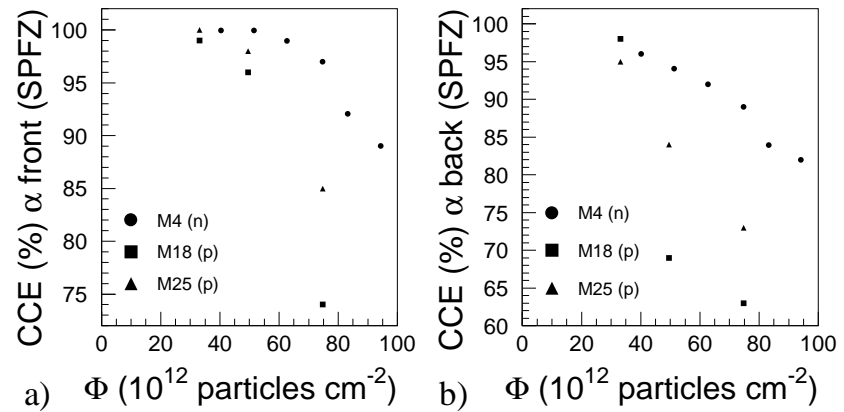

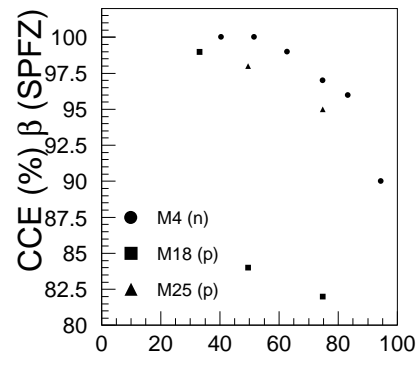

c) $\Phi\left(10^{12}\right.$ particles $\left.\mathrm{cm}^{-2}\right)$

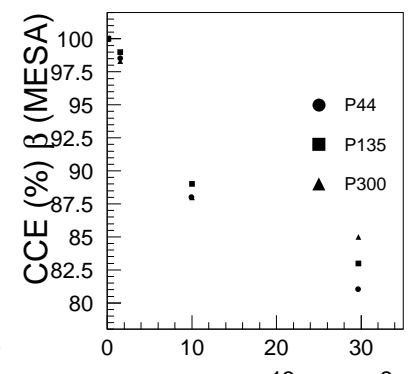

d)

$\Phi\left(10^{13} \mathrm{p} \mathrm{cm}^{-2}\right)$

Figure 14: Charge collection efficiency as a function of fluence using: a) $\alpha$ particles incident on the front, b) $\alpha$ particles incident on the back and c-d) $\beta$ particles.

fluences of $\approx 10^{14}$ particles $/ \mathrm{cm}^{2}$. The model was also adapted to be used with MESA diode by considering one dead layer on each side of the diode and a delayed component.

For both types of detectors (SPFZ and MESA), this model gives mobilities decreasing linearily up to fluences of around 5 . $10^{13}$ particles $/ \mathrm{cm}^{2}$ and beyond, converging to saturation values of about $1000 \mathrm{~cm}^{2} / \mathrm{Vs}$ and $455 \mathrm{~cm}^{2} / \mathrm{Vs}$ for electrons and holes, respectively.

At a fluence $\Phi \approx 10^{14}$ particles $/ \mathrm{cm}^{2}$, the charge carrier lifetime degradation due to trapping with increased fluence is responsible for a charge collection deficit of about $13 \%$ for $\beta$ particles, $25 \%$ for $\alpha$ particles incident on the front side and 35 $\%$ for $\alpha$ particles incident on the back side of SPFZ and MESA diodes, which is in agreement with direct measurements.

\section{REFERENCES}

[1] H. K. Gummel, A self-consistent iterative scheme for onedimensional steady state transistor calculations, IEEE Trans. Electron. Devices ED-11 (1964) 455.

[2] S. Ramo, Currents induced by electron motion, Proc. IRE vol. 27(9) (1939) 584.

[3] S.M. Sze, Semiconductor devices, Physics and Technology, AT\&T Bell Laboratories, Murray Hill, New Jersey, 1965.

[4] D.M. Caughey and R.E. Thomas, Carrier mobilities in silicon empirically related to doping and field, Proc. IEEE 55 (1967) 2192.

[5] Application Software Group, MINUIT Function Minimization and Error Analysis Reference Manual, Version 92.1, i, (1992). 
[6] Z. Li and H.W. Kraner, Fast neutron radiation damage effects on high resistivity silicon junction detectors, Journ. of Elec. Mat. vol. 21(7) (1992) 701.

[7] L.J. Beattie et al., The electric field in irradiated silicon detectors, ATLAS note INDET-NO-194, 9 Dec. 1997.

[8] D. Menichelli et al., Modelling of observed double junction effect, International Conference on Radiation Effects on Semiconductor Materials, Detectors and Devices, Florence, Italy, March 1998, to be published in Nucl. Instr. and Meth. A.

[9] G. Casse et al., Impact of mesa and planar processes on radiation hardness of Si detectors, CERN-ECP/97-09, January 1998.

[10] S. Pospísil, Scanning of silicon detector using alpha particles and low energy protons, talk given at GaAs'98, Pruhonice, 22-26 june 1998, to be published in Nucl. Instr. and Meth. A (1998).

[11] C. Leroy et al., Study of electrical properties and charge collection of silicon detectors under neutron, proton and gamma irradiations, Proc. IVth Int. Conf. on Calorimetry in High Energy Physics, World Scientific, eds. A. Menzione and A. Scribano, Singapore (1994) 627. 